\title{
Mixture models applied to heterogeneous populations
}

\author{
Carolina V. Cavalcante $^{\mathrm{a}}$ and Kelly C. M. Gonçalves ${ }^{\mathrm{b}}$ \\ ${ }^{a}$ Escola Nacional de Ciências Estatísticas (ENCE) \\ ${ }^{\mathrm{b}}$ Universidade Federal do Rio de Janeiro (UFRJ)
}

\begin{abstract}
Mixture models provide a flexible representation of heterogeneity in a finite number of latent classes. From the Bayesian point of view, Markov Chain Monte Carlo methods provide a way to draw inferences from these models. In particular, when the number of subpopulations is considered unknown, more sophisticated methods are required to perform Bayesian analysis. The Reversible Jump Markov Chain Monte Carlo is an alternative method for computing the posterior distribution by simulation in this case. Some problems associated with the Bayesian analysis of these class of models are frequent, such as the so-called "label-switching" problem. However, as the level of heterogeneity in the population increases, these problems are expected to become less frequent and the model's performance to improve. Thus, the aim of this work is to evaluate the normal mixture model fit using simulated data under different settings of heterogeneity and prior information about the mixture proportions. A simulation study is also presented to evaluate the model's performance considering the number of components known and estimating it. Finally, the model is applied to a censored real dataset containing antibody levels of Cytomegalovirus in individuals.
\end{abstract}

\section{Introduction}

Mixture models are noted for their flexibility in modeling complex data and are widely used in the statistical literature (see McLachlan and Peel (2004)). These models provide a natural framework for modeling heterogeneity in a population. Moreover, due to the large class of functions that can be approximated by mixture models, they are attractive for describing non-standard distributions and have been adopted in many areas, such as genetics, ecology, computer science, economics, biostatistics and many others. For instance, as stated in Jordan (2004), in genetics, location of quantitative traits on a chromosome and interpretation of microarrays are both related to mixtures, while, in computer science, spam filters and web context analysis start from a mixture assumption to distinguish spams from regular emails and to group pages by topic, respectively.

Statistical analysis of mixtures is not straightforward and the Bayesian paradigm has been particularly suited for this purpose. This framework allows the compli-

Key words and phrases. Identifiability, sensitivity analysis, subpopulations, frequentist properties, NHANES.

Received October 2015; accepted November 2016. 
cated structure of a mixture model to be decomposed into a set of simpler structures through the use of hidden or latent variables. According to Richardson and Green (1997), when the number of components is unknown, the Bayesian paradigm is the only sensible approach to its estimation. Thus, the Bayesian approach has enabled mixture models to become increasingly popular in many areas. In real applications, the number of components can have important implications regarding the problem, so it has to be well specified or estimated, although practitioners usually have little theoretical guidance. On the other hand, even if prior theory suggests a particular number of components, it may not be possible to reliably distinguish between some of the components. In some cases additional components may simply reflect the presence of outliers in the data.

When the number of subpopulations is assumed to be known, Markov Chain Monte Carlo methods (MCMC) can be used for Bayesian estimation of the subpopulation parameters. Nevertheless, this method, as originally formulated, requires the posterior distribution to have a density with respect to some fixed measure. When the number of components is considered unknown, that is, the size of the parameter space is also a parameter, a problem with variable dimension appears, so MCMC cannot be used alone and more sophisticated methods are required to perform the Bayesian analysis. One alternative in this case is the approach based on Reversible Jump MCMC (RJMCMC), which was first proposed by Green (1995) and applied in univariate normal mixture models with unknown numbers of components by Richardson and Green (1997). The method basically consists of jumps between the parameter subspaces corresponding to different numbers of components in the mixture.

While MCMC provides a convenient way to draw inference from complicated statistical models, there are still many, perhaps under appreciated, problems associated with the MCMC analysis of mixtures. These problems are mainly caused by the nonidentifiability of the components under symmetric priors, which leads to the so called label-switching problem in the MCMC output, discussed in Jasra, Holmes and Stephens (2005). The term describes the invariance of the likelihood function under relabelling of the mixture components, which can cause the posterior distribution of the parameters to be highly symmetric and multimodal. Therefore, the component labels are mixed up and cannot be distinguished from each other. As a result, the usual practice of summarizing joint posterior distributions by marginal distributions, and for instance, estimating parameters by their posterior mean is often inappropriate, because the marginal on the parameters for all components is identical and the posterior expectation for the parameters is identical too. A frequent response to this problem is to remove the symmetry by using artificial identifiability constraints. This and other alternative approaches to this problem are described by Stephens (2000). 
The aim of this work is to review and discuss some aspects in the inference of mixture models, in particular the normal mixture models, when applied to heterogeneous populations under the Bayesian approach. The main purpose is to evaluate the model's performance in different settings of heterogeneity, prior information for mixture weights and when considering the number of components known or unknown.

Most previous works, as Jasra, Holmes and Stephens (2005), in general, evaluate a possible improvement in the label-switching problem choosing a more appropriate relabelling algorithm. However, the aim here is to verify if the labelswitching phenomena generally persists when the subpopulations are not well separated and how the level of heterogeneity of the population affects the results. Furthermore, we evaluate the label-switching phenomenon by assuming more informative prior distributions for the mixture weights and show that in some cases it can be more efficient than use different relabelling algorithms known in the literature.

Although works as Nobile (2004) and Richardson and Green (1997) carried out some studies about the influence of hyperparameters specification on posterior distribution of other parameters, different from this paper, they did not connect the prior distribution of the weights with the label-switching problem, which is our main result.

On the other hand, previous works that compare the results obtained under RJMCMC with MCMC, as Richardson and Green (1997) for the univariate case and Dellaportas and Papageorgiou (2006) for the multivariate one, use as a diagnostic tool mainly the predictive density and the posterior estimates of $k$. In this work, we also evaluate the model's performance under a simulation study in order to verify frequentist properties of the Bayes' estimators produced from each method.

The paper is organized as follows. Section 2 presents the general definition of a mixture model and discusses some aspects of inferences. A simulation study for assessing the estimation of model parameters under different levels of heterogeneity is presented in Section 3. Additionally, a prior sensitivity analysis of the mixture proportions is presented in order to see its effect under label-switching phenomena. We also discuss the model fit when the number of components is known and unknown. In Section 4, the performance of the method is assessed through an application to a left-censored real dataset. Thus, we discuss briefly the inference in this case. Finally, Section 5 presents some conclusions and suggestions for further research.

We particularly used the R Core Team (2014) package called mixAK, proposed by Komárek (2009), with routines to compute the posterior distribution through MCMC and RJMCMC for multivariate right, left and interval-censored observations. Appendix A presents the main code that can be used to perform the experiments presented here. 


\section{Finite mixture models}

The basic mixture model for independent scalar or vector observations $Y_{i}, i=$ $1, \ldots, n$, is a convex combination given by:

$$
Y_{i} \sim \sum_{j=1}^{k} w_{j} f\left(\cdot \mid \boldsymbol{\theta}_{j}\right), \quad i=1, \ldots, n,
$$

where $f(\cdot \mid \boldsymbol{\theta})$ is a given parameter family of densities indexed by a scalar or a vector $\boldsymbol{\theta}$. In general, the objective of the analysis is to make inferences about the unknowns: the number of components, $k$; the parameters $\boldsymbol{\theta}=\left(\boldsymbol{\theta}_{1}, \ldots, \boldsymbol{\theta}_{k}\right)$ with $\boldsymbol{\theta}_{j}$ being specific to component $j$; and the components' weights, $\mathbf{w}=\left(w_{1}, \ldots, w_{k}\right)$, $0<w_{j}<1, \sum_{j=1}^{k} w_{j}=1$. Let $\boldsymbol{\Phi}=(\mathbf{w}, \boldsymbol{\theta}, k)$ be the parametric vector of the model (2.1).

For an observed random sample $\mathbf{y}=\left(y_{1}, y_{2}, \ldots, y_{n}\right)^{\prime}$, the likelihood function of $\boldsymbol{\Phi}$ is given by:

$$
p(\mathbf{y} \mid \boldsymbol{\Phi})=\prod_{i=1}^{n} \sum_{j=1}^{k} w_{j} f\left(y_{i} \mid \boldsymbol{\theta}_{j}\right) .
$$

The likelihood function leads to $k^{n}$ terms, which creates computational difficulty.

A context in which the model (2.1) can arise and we are interested in this paper is when we postulate a heterogeneous population consisting of heterogeneous groups $j=1,2, \ldots, k$ of sizes proportional to $w_{j}$, from which a random sample is drawn. The label of the group from which each observation is drawn is unknown, so it is natural to regard the group label $z_{i}$, for the $i$ th observation as a latent variable and rewrite (2.1) as the following hierarchical model: for $i=1, \ldots, n$, $j=1, \ldots, k$,

$$
Y_{i} \mid \boldsymbol{\theta}_{j}, z_{i}=j \sim f\left(\cdot \mid \boldsymbol{\theta}_{j}\right), \quad \text { with } P\left(z_{i}=j\right)=w_{j} .
$$

By integrating $\mathbf{z}=\left(z_{1}, \ldots, z_{k}\right)$ out from (2.3), we return to model (2.1). The formulation given by (2.3) is convenient for interpretation and calculation. Latent indicator variables usually leads to an efficient simulation algorithm that quickly focuses on the modes of the posterior distribution, thus it will contribute to reducing the computational effort.

A Bayesian approach to inference requires the specification of a prior distribution $p(\cdot)$ for the parameters of the mixture model (2.1). In particular, prior elicitation is an important step. According to Roeder and Wasserman (1997), in a mixture content, assuming a non-informative prior yield improper posterior distributions. Since there is always the possibility that no observations are allocated to one or more components, standard choices of independent improper non-informative prior distributions for the component parameters cannot be used. Richardson and Green (1997) proposes an alternative in this case based on keeping 
the simple independence and define weakly informative priors, which may or not be data dependent.

The mixture model in (2.3) is invariant to permutation of the labels $j=1, \ldots, k$. Some implications of this for likelihood analysis are discussed by Redner and Walker (1984). If we have no prior information that distinguishes between the components of the mixture, so the prior distribution $p(\cdot)$ is the same for all permutations of $\boldsymbol{\theta}$, then the posterior distribution will also be symmetric and, there will be $k$ ! symmetric modes in the posterior distribution. Then, the marginal posterior distributions for the parameters will be identical for each mixture component and the posterior means of all the components are the same, thus they are poor estimates of these parameters. Thus, if the problem is not handled properly, the ergodic average of the MCMC samples is not appropriate for the estimation of the parameters.

There are some suggested solutions to this problem, see Stephens (2000) for details. One common response to the label-switching problem is to impose an identifiability constraint on the parameter space. This breaks the symmetry of the prior and thus, of the posterior distribution of the parametric vector. For example, we can impose an ordering constraint on $\theta_{j}$ 's, such as $\theta_{1}<\theta_{2}<\cdots<\theta_{k}$, if it is a scalar.

\subsection{Inference}

Since we are in a Bayesian framework, the inference consists of obtaining the posterior distribution of the parametric vector $\boldsymbol{\Phi}$ of model (2.3). In general, this joint distribution cannot be obtained in closed form. One alternative, which is often used and is feasible to implement, is to generate samples from the marginal distributions of the parameters based on the MCMC algorithm. A comprehensive Bayesian treatment using MCMC methods was presented in Diebolt and Robert (1994) for finite mixture models.

Nevertheless, this method, as originally formulated, requires the posterior distribution to have a density with respect to some fixed measure. Thus, in the mixture context, the method can only be applied when the number of components $k$ in the model (2.3) is considered known.

However, the number $k$ is rarely known, and setting an incorrect value can bring important consequences to the posterior distribution. For instance, on an extremal case, a mixture model with only one component will roughly impose zero density to some ranges of the density, while a mixture with the number of components equal to the number of observations, simply will over-fit the data, creating unnecessary clusters. Thus, a halfway solution offers a trade off between these two solutions, providing a good fit of the data, modeling well all ranges of the density, without a wrong large number of parameters to estimate. Other times, the target of the study is exactly the estimation of $k$. The approach based on RJMCMC is an alternative in this case, as proposed in this context by Richardson and Green (1997). 
It operates on the augmented parameter space, where the allocation variables $\mathbf{z}$ are included as unknown parameters.

The method basically consists of jumps between the parameter subspaces corresponding to different numbers of components in the mixture, after updating them. If the current model is a mixture with $k>1$ components, then it is usual to reduce the searching strategy to moves that either preserve the number of components, or lead to a mixture with $k-1$ or $k+1$ components. The idea is then to supplement each of the spaces with adequate artificial spaces in order to create a bijection between them, most often by augmenting the space of the smaller model. Jumps are achieved by adding new components, deleting existing components, and splitting or merging these. These moves are randomly chosen and after being drawn, it is necessary to make corresponding changes to $(\boldsymbol{\theta}, \mathbf{w})$.

One can assess convergence for each mixture component parameters, however, as mentioned before, label-switching issues lead to a type of poor mixing in the mixture component-specific parameters, which may not impact convergence and mixing of the induced predicitive density. In particular, due to exchangeability of the mixture components, its marginal posterior distributions is identical for all subgroups and hence the chains for each of the mixture component-specific parameters have the same target distribution. For instance, in a normal mixture model with $k=2$ components, suppose one mixture component located at $\mu_{1}=0$ and the other at $\mu_{2}=-1$. The Gibbs sampler for $\mu_{1}$ should then randomly jump between values close to 0 and values close to -1 if mixing is good. The posterior for $\mu_{i}, i=1,2$ has a multimodal form with one mode close to 0 and one mode close to -1 . If these modes are well separated and there is a region of low probability density between the modes, then the Gibbs sampler will remain stuck for long intervals in one mode. A well-mixing Gibbs sampler should switch between these modes often, but in practice for well separated components, it is common to remain stuck in one labeling across all the samples are collected.

For those reasons, it is not appropriate to simply calculate posterior summaries based on the posterior draws of the parameters. In the two-component illustration, one would obtain the same posterior mean for $\mu_{1}$ and $\mu_{2}$ if the Gibbs sampler mixed well enough and sufficiently many samples were drawn. If we could relabel the samples so that after relabelling all the samples of $\left(\mu_{1}, \sigma_{1}^{2}\right)$ correspond to the component at $\mu=0$ and all the samples of $\left(\mu_{2}, \sigma_{2}^{2}\right)$ correspond to the component at $\mu=-1$, then we could calculate posterior summaries of the mixture component-specific parameters in the standard manner. This relabelling can be done using postprocessing algorithms or with constraints in the prior in an attempt to make the separate mixture components distinguishable. In this paper, we will restrict imposing an increasing order to the mixture means in the prior distribution, so that the higher indexed components have higher means. 
However, for any given dataset, many identifiability constraint choices can be ineffective in removing the symmetry from the posterior distribution. For instance, in multivariate cases it is not at all clear in most cases what type of restriction is appropriate. Even in the univariate case, it may be that we need multiple components with similar means but different variances to provide a good fit to the data. If the means are close together, then label-switching can occur even if we place a strict order restriction on the means and hence the restriction does not fully solve the label ambiguity problem.

\subsection{Normal mixture model}

In this work, we are particularly interested in the univariate normal case presented in Richardson and Green (1997), so $\boldsymbol{\theta}_{j}$ in (2.1) becomes a vector with expectation and variance parameters $\left(\mu_{j}, \sigma_{j}^{2}\right)$. The model is stated below: for $i=1, \ldots, n$ and $j=1, \ldots, k$,

$$
\begin{gathered}
Y_{i} \mid \mu_{j}, \sigma_{j}^{2}, z_{i}=j \sim \operatorname{Normal}\left(\mu_{j}, \sigma_{j}^{2}\right), \\
P\left(z_{i}=j\right)=w_{j} .
\end{gathered}
$$

Assuming that the parameters in $\boldsymbol{\Phi}$ are prior independent and identically distributed and that $k$ is unknown, the prior distribution is given by: for $i=1, \ldots, n$ and $j=1, \ldots, k$,

$$
\begin{aligned}
\mathbf{w} & \sim \operatorname{Dirichlet}(\gamma), \\
\mu_{j} & \sim \operatorname{Normal}\left(\mu_{a}, \sigma_{a}^{2}\right), \quad j=1, \ldots, k, \\
\sigma_{j}^{-2} & \sim \operatorname{Gamma}(\alpha, \beta), \quad j=1, \ldots, k, \\
\beta & \sim \operatorname{Gamma}(g, h), \\
k & \sim \operatorname{Uniform}\left\{1, k_{\max }\right\},
\end{aligned}
$$

where $\operatorname{Dirichlet}(a)$ generically denotes the symmetric Dirichlet distribution with parameter $a$. The symmetric Dirichlet distributions are often used, since there typically is no prior knowledge favoring one component over another. Since all elements of the parameter vector have the same value, the distribution is alternatively parameterized by a single scalar value $a$. $\operatorname{Gamma}(a, b)$ represents the gamma distribution with mean $a / b$ and variance $a / b^{2}$ and Uniform $\{a, \ldots, b\}$ is the uniform distribution defined on the integers $\{a, \ldots, b\}$. Moreover, for identifiability, we can use for example that the $\mu_{j}$ are in increasing numerical order, thus the joint prior distribution of $\boldsymbol{\Phi}$ is $k$ ! times the product of their marginal prior distributions.

In this paper, as done in Richardson and Green (1997) we consider the Bayesian estimation in the set-up where we do not have strong prior information about the mixture parameters. Thus, we use weakly informative priors and the default hyperparameter choices can be seen with further details in Richardson and Green (1997). 
As mentioned in Section 2.1, in this work the unique labeling is achieved by imposing a restriction on $\mu_{j}$. We use that in which the $\mu_{j}$ are in increasing numerical order; so the joint prior distribution of the parameters is $k$ ! times the prior density, restricted to the set $\mu_{1}<\mu_{2}<\cdots<\mu_{k}$. When the means are well separated, labels of the realizations from the posterior by ordering their means generally coincide with the population ones. As the separation gets small, label-switching can occur. This problem can be also minimized by choosing to order other parameters of the mixture components, for example, the variance, weights or some combination of all three parameters.

\section{Simulation study}

To assess the convergence of the MCMC and RJMCMC estimation, we generated some samples under different settings. We obtained samples from the posterior distributions of the model parameters, supposing $k$ known and estimating it. The population estimates were then compared with the true values to evaluate the model's performance. The aim was to evaluate the performance of the normal mixture model varying the level of heterogeneity and the prior information elicited for the mixture proportions. Furthermore, we also compared the results obtained under each simulation method considered, MCMC and RJMCMC.

\subsection{Assessment of RJMCMC and MCMC under different scenarios}

To check the convergence of the RJMCMC and MCMC estimations, we generated one sample with $n=100$ observations under two levels of heterogeneity, the first one with well-separated groups, which we call the more heterogeneous sample, and the other with groups less well separated, which represents the more homogeneous one. In both scenarios, we fixed $k=5, \sigma^{2}=\left(\sigma_{1}^{2}, \ldots, \sigma_{5}^{2}\right)=$ $(0.22,1.95,0.92,0.74,1.13)$ and $\mathbf{w}=\left(w_{1}, \ldots, w_{5}\right)=(0.17,0.21,0.34,0.12$, 0.16). With these values fixed for $\mathbf{w}$, we expected to have groups with a reasonable number of observations, so we did not consider scenarios with groups outliers. The heterogeneous scenario was obtained by fixing $\boldsymbol{\mu}=\left(\mu_{1}, \ldots, \mu_{5}\right)=$ $(-3,0,4,11,16)$ and the homogeneous by setting $\boldsymbol{\mu}=\left(\mu_{1}, \ldots, \mu_{5}\right)=(0,2,4$, $6,8)$. Figure 1 presents the distribution of both datasets generated. The aim of this study is to verify how the level of heterogeneity of the population affects the results, mainly regarding the label-switching problem.

The prior distribution considered are described in (2.5), and we elicited the prior for $\mu_{j}$ and $\sigma_{j}^{2}$ using the same idea of weakly informative prior suggested by Richardson and Green (1997). Moreover, we sort all components according to increasing order of magnitude of the posterior means to avoid the label switching phenomenon. 


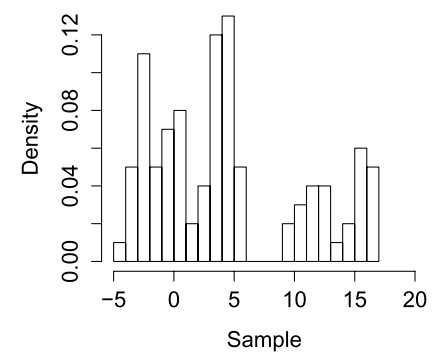

(a) Heterogeneous

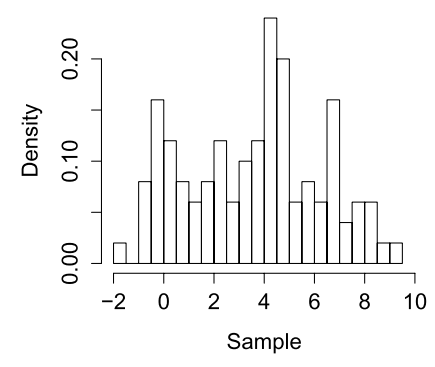

(b) Homogeneous

Figure 1 Histograms with the distribution of the samples generated.

First, we assumed $k$ unknown and in its prior distribution presented in (2.5) we assumed $k_{\max }=10$, thus RJMCMC was used to obtain samples from the posterior distribution. We also did a brief prior sensitivity analysis assuming two values of the Dirichlet prior distribution for each dataset: $\gamma \in\{1,4\}$ for the heterogeneous case and $\gamma \in\{1,4,10\}$ for the homogeneous case. To assume $\gamma=1$ is equivalent to assuming a uniform distribution over all points in its support. On the other hand, the parameter value above 1 gives some information that all sample proportions in subpopulations are similar to each other.

For the RJMCMC simulations, we generated, respectively for the homogeneous and heterogeneous cases, 350,000 and 70,000 samples from the posterior distribution, discarded the first 10,000 and 20,000, and then thinned the chain by taking every 10th sample value. Figure 2 displays the histogram with the posterior densities of $k$ for some values of $\gamma$. It should be noted that for the heterogeneous case the parameter $k$ is well estimated, but when $\gamma=4$, the estimate is more accurate. On the other hand, $k$ is underestimated when assuming $\gamma=4$ with the homogeneous sample. The same happens with $\gamma=1$ or any value less than 4 . In this case, when $\gamma=10$ the value of $k$ is well estimated.

Figure 3 shows the trace plot with the posterior distribution of parameters $\mu_{j}$ conditional on the posterior samples, whose estimated value of $k$ is the one with highest posterior probability. Here we also considered the value of $k$ known and fixed it as the true value used to generate the samples, so MCMC was also used to generate samples from the posterior distribution. For the MCMC simulations, we generated 70,000 samples from the posterior distribution, discarded the first 20,000 , and then thinned the chain by taking every 10th sample value, for both datasets generated.

All the results were obtained for each scenario and value of $\gamma$ considered. The black trace represents the posterior density when setting $\gamma=1$, the blue trace when $\gamma=4$ in both scenarios and $\gamma=10$ is represented by the red trace in the homogeneous case. The gray line represents the true value of each $\mu_{j}$. Note that in the homogeneous case, when RJMCMC is used, there is only a red trace for $\mu_{5}$. 

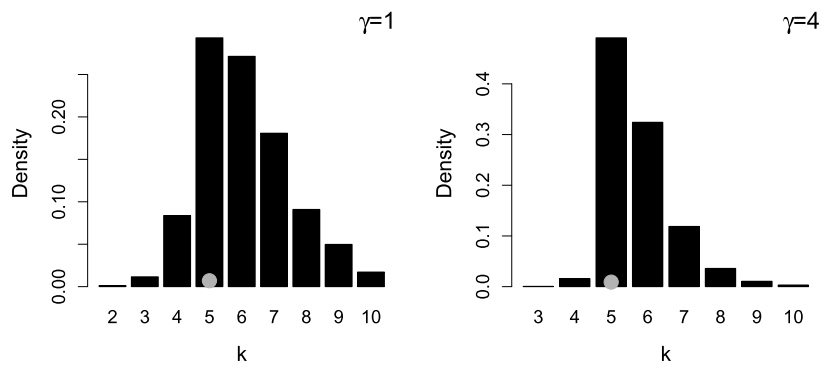

(a) Heterogeneous
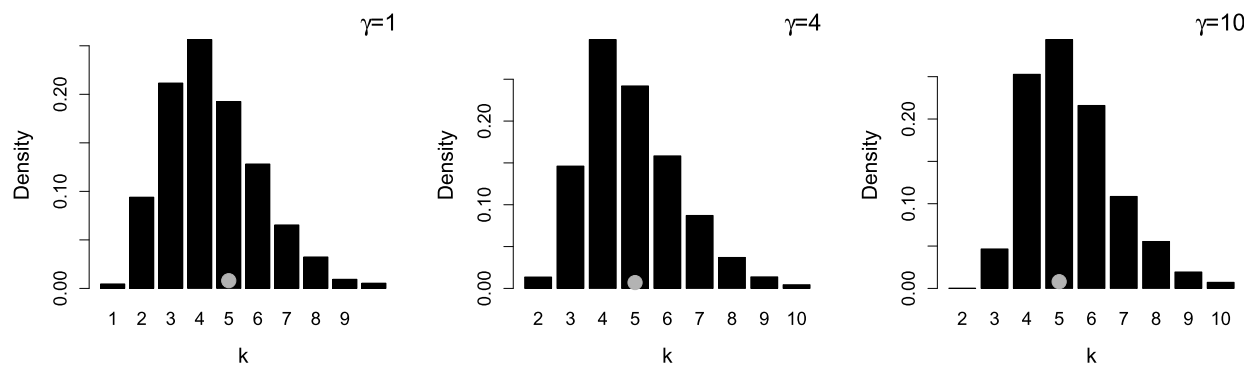

(b) Homogeneous

Figure 2 Posterior densities of the parameter $k$ for each value of $\gamma$ considered in the prior distribution of the mixture proportions. The gray point represents the true value fixed in the simulation.

The reason is that the posterior for $k$ favors the value 5 only when $\gamma=10$. When analyzing Figure 3, we can see that the raw samples jump around between different symmetric regions in the trace plots, which explains the multimodalities of the marginal densities for raw samples. Thus, the effects of label-switching can be seen in the sampled values of the component means for many cases, even in the heterogeneous case. However, this behavior improves when giving some prior information about the mixture proportions. It is also possible to observe that in the homogeneous case it is necessary to increase the value of $\gamma$ even more, that is, to give more prior information that the proportions observed in groups are similar, in order to minimize the label-switching effects and attain the convergence.

The results obtained indicate that the RJMCMC and MCMC chains converged in some cases, but in others the label-switching phenomenon appears significantly, so estimating the means on the basis of the RJMCMC and MCMC output is not straightforward. However, as the value of $\gamma$ increases this behavior improves. If the number of iterations increases, and so does the lag of the chain, the convergence can also improve, but this would require high computational effort. Thus, we suggest careful elicitation of the prior distribution to have better estimates. Almost all the mean parameters are well estimated when $\gamma=4$ and $\gamma=10$ in the heteroge- 

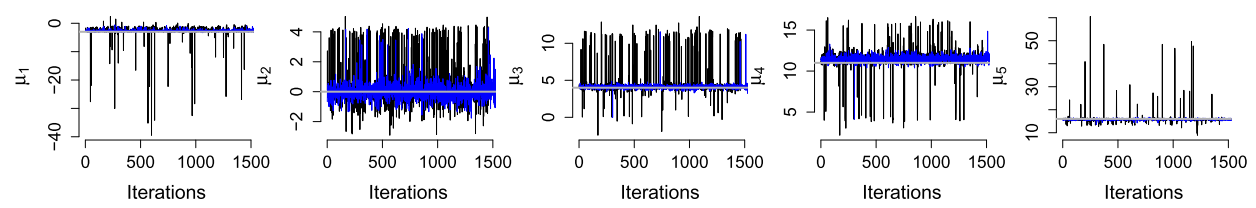

(a) Heterogeneous - RJMCMC
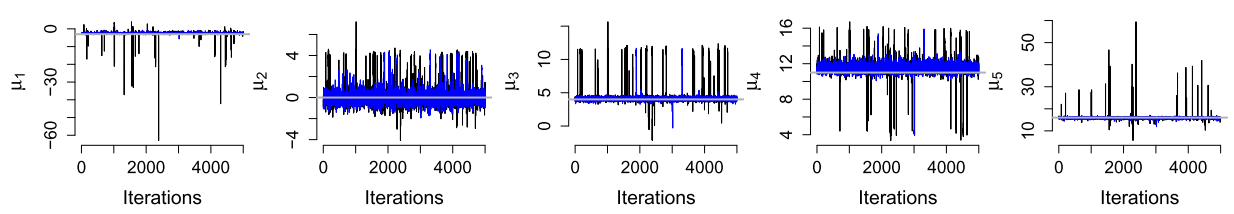

(b) Heterogeneous - MCMC

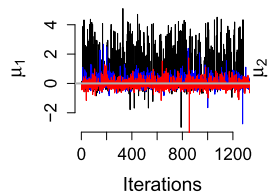

Iterations
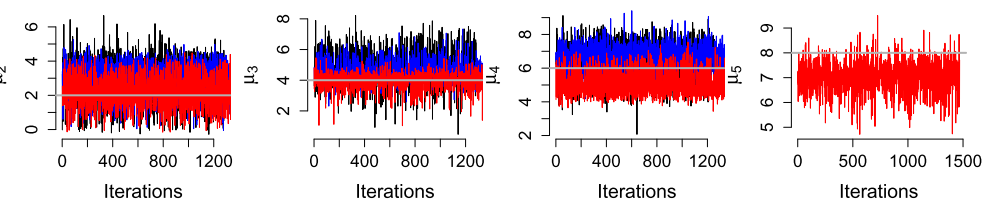

(c) Homogeneous - RJMCMC
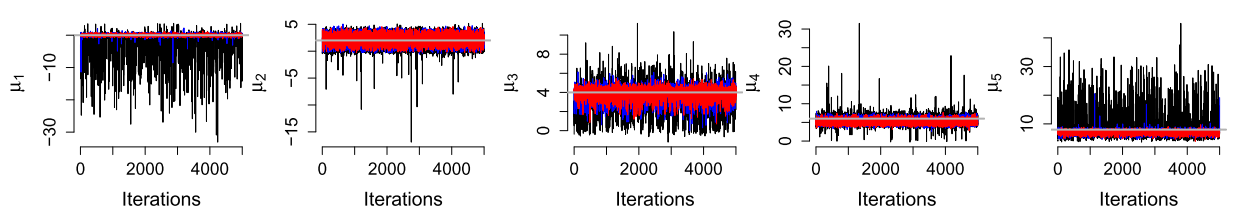

(d) Homogeneous - MCMC

Figure 3 Trace plots with the posterior densities of the parameters of $\boldsymbol{\mu}$ obtained from fitting the normal mixture model under the different priors considered for $\mathbf{w}$ and the two samples. We also assumed the value of $k$ to be unknown (RJMCMC) and known (MCMC). The black trace is obtained assuming $\gamma=1$, the blue one when $\gamma=4$ and the red with $\gamma=10$. The gray line represents the true value of each $\mu_{j}, j=1, \ldots, 5$.

neous and homogeneous case, respectively. The traces and density estimates for the mixture proportions and variances present this same behavior.

In general, MCMC and RJMCMC present similar behavior, mainly for the heterogeneous sample generated. A more interesting comparison between the two approaches is presented in the next subsection.

Figure 4 shows summary statistics of the posterior distributions of the mean parameters after reaching the supposed convergence for each of the scenarios and prior assumed, when assuming $k$ unknown. The crosses represent the true value, the lines the $95 \%$ credibility interval and the points are the posterior mean. Also, the results in black are obtained assuming $\gamma=1$, the blue one when $\gamma=4$ and the red when $\gamma=10$. In almost all the cases, the intervals contain the true value. It is 


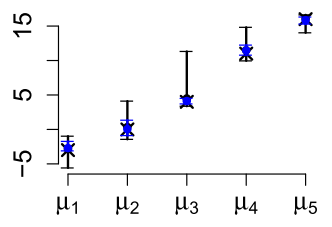

(a) Heterogeneous

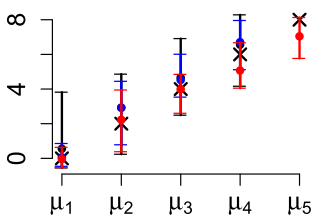

(b) Homogeneous

Figure 4 Summary measurements for the point and 95\% credibility interval estimates of the mean parameters model for a heterogeneous and homogeneous sample under three prior distributions for w: the results in black are obtained assuming $\gamma=1$, the blue one when $\gamma=4$ and the red when $\gamma=10$. Here it was considered the value of $k$ unknown, so RJCMC was used. The crosses represent the true value, the lines the $95 \%$ credibility interval and the points are the posterior mean.

possible to observe the impact of the label-switching, which hampers estimating the parameters, but also the improvement in the results when assuming a more informative prior to $\mathbf{w}$.

Therefore, we conclude that in the cases considered here, the identifiability problem can be minimized under more informative priors and it is not necessary to use other alternative approaches to deal with the identifiability problem, like those described in Jasra, Holmes and Stephens (2005). Although we named that as an informative prior distribution for the mixture weights, its elicitation does not necessarily need prior information about the weights. In practice, we elicited its hyperparameters based on the MCMC performance and the distribution of the dataset among the groups.

The prior distribution of $\mathbf{w}$ seems to have strong impact on the posterior distribution, improving the results, even in the homogeneous case. Furthermore, as the degree of heterogeneity increases, the mixture model's performance improves considerably even under less prior information. The same conclusions were attained when estimating the value of $k$ or considering it known.

Additionally, Figure 5 shows the predictive densities for the two datasets generated, for all the prior distributions considered for $\mathbf{w}$, represented by the solid $(\gamma=1)$, dashed $(\gamma=4)$ and dotted $(\gamma=10)$ lines, respectively. The predictive densities in black are those obtained when the value of $k$ is estimated, so RJM$\mathrm{CMC}$ was used, and the red ones are obtained when the value of $k$ is fixed at the true value, so MCMC was used. The densities obtained under RJMCMC are conditional on the posterior samples, whose sampled value of $k$ is equal to the value with highest posterior probability among all the samples. In contrast with the above results, an estimate of the predictive density based on the RJMCMC and MCMC outputs is unaffected by the label-switching problem, since it does not depend on how the components are labeled. The predictive density is better estimated in the heterogeneous sample than the homogeneous one and the prior distribution does not affect the estimates. Moreover, the results obtained in the estimation considering $k$ unknown and fixed are very similar to each other. 


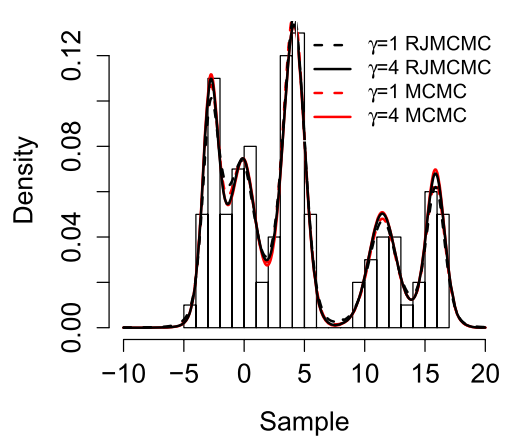

(a) Heterogeneous

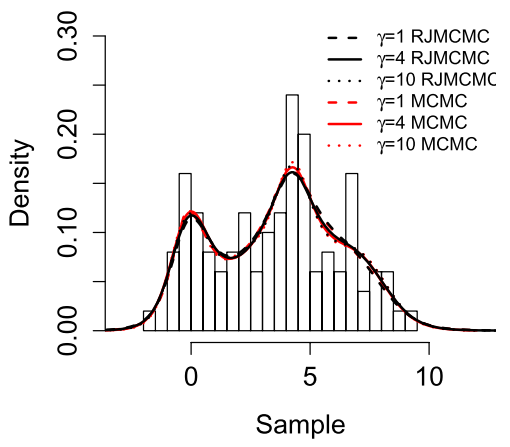

(b) Homogeneous

Figure 5 Predictive densities considering different prior distributions for $\mathbf{w}$ and estimating the value of $k(R J M C M C)$ and fixing it on its true value (MCMC).

\subsection{Comparison between RJMCMC and MCMC}

To examine the performance of the Bayesian estimators obtained under each simulation method, we generated two artificial samples of size $n=100$, fixing $k$ at two different values, $k=3$ and $k=5$, in order to evaluate the results when varying the value of $k$. Then, we obtained samples from the posterior distribution of the parametric vector, assuming $k$ known (MCMC) and estimating it (RJMCMC). In the MCMC simulation we particularly set $k$ for each case at three different values: we assumed it to be 2, 3 and 4 for the first sample and 4, 5 and 6 for the second one. We assumed here the same prior distribution used in Section 3.1. Thus, we are interested in evaluating the method's performance when we fix $k$ as its true value, or a smaller or a greater value than the true one, and when it is estimated. For the RJMCMC and MCMC simulations, we generated 70,000 samples from the posterior distribution, discarded the first 10,000, then thinned the chain by taking every 10th sample value, and the convergence was achieved.

Figure 6 presents the posterior distribution of $k$ obtained in the RJMCMC simulation and the predictive densities obtained for each sample generated. It should be noted that $k$ is well estimated and all predictive densities are very similar, except when we fixed $k$ lower than the true value. Moreover, setting $k$ higher than the true value does not affect the results.

Selecting a suitable number of mixture components if it is not known in advance, coincides with the problem of model selection. A general approach to comparison of complex models based on the samples from the posterior distribution has been suggested by Spiegelhalter et al. (2002) who introduced the Deviance Information Criterion (DIC). Nevertheless, its use in mixture models is controversial, because the posterior expectation is not a suitable plug-in estimate for the model parameters since it lies in between multiple modes of the posterior density, 

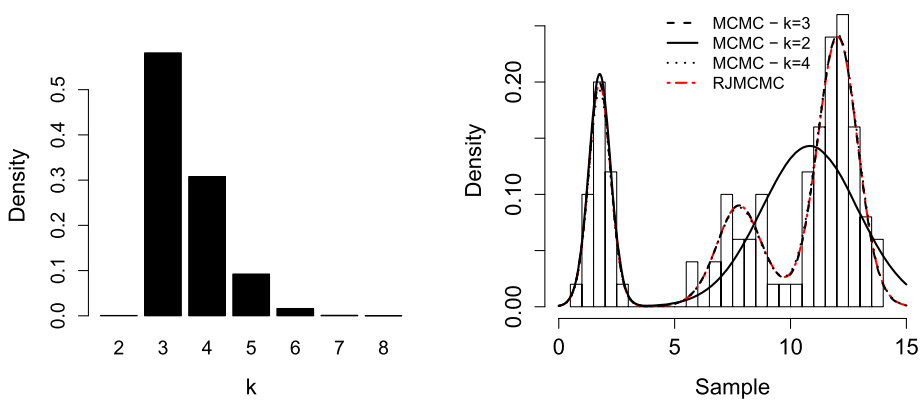

(a) $k=3$
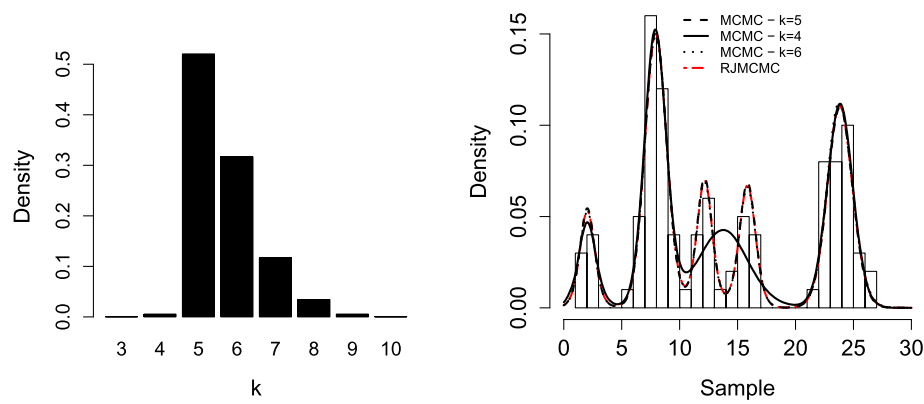

(b) $k=5$

Figure 6 Predictive densities considering $k$ known (MCMC) and fixing it at $k, k-1, k+1$ and estimating it (RJMCMC).

and alternative plug-in estimators are hard to define. Other versions of DIC for mixture and in general missing data models have been discussed by Celeux et al. (2006). DIC is computed as DIC $=\bar{D}+p_{D}$, such as $p_{D}=\bar{D}-\tilde{D}$, where $\bar{D}$ is the approximation to the posterior mean of the deviance, $\tilde{D}$ is the deviance evaluated in the "estimate" to the model parameters and $p_{D}$ is the effective dimension of parameters.

Plummer (2008) suggested to use penalized loss function for Bayesian model comparison and showed that DIC is an approximation to a penalized loss function based on the deviance, with a penalty derived from a cross-validation argument. Particularly in mixture context, Plummer (2008) recommends to use the Penalized Expected Deviance (PED), which is computed as PED $=\hat{D}_{e}+\hat{p}_{\text {opt }}$, where $\hat{D}_{e}$ is the estimated expected deviance, where the estimate is based on two parallel chains, $\hat{p}_{\text {opt }}$ is the estimated penalty, where the estimate is based on simple MCMC average based on two parallel chains.

Tables 1 and 2 shows the value of $\mathrm{DIC}_{3}$ presented in Celeux et al. (2006) and PED, respectively, for each approach considered in this study. As both evaluates the goodness of fit of the model, so the model with the smallest DIC and PED 
Table 1 DIC measurements for each model considered

\begin{tabular}{lccccccr}
\hline & \multicolumn{3}{c}{$k=3$} & & \multicolumn{3}{c}{$k=5$} \\
\cline { 2 - 3 } & DIC & $\bar{D}$ & $p_{D}$ & & DIC & $\bar{D}$ & $p_{D}$ \\
\hline RJMCMC & 438.91 & 431.83 & 7.08 & & 557.26 & 546.30 & 10.96 \\
MCMC $(k-1)$ & 518.09 & 497.03 & 21.06 & & 571.38 & 563.39 & 7.99 \\
MCMC $(k)$ & 437.60 & 431.15 & 6.45 & & 555.34 & 545.35 & 9.99 \\
$\operatorname{MCMC~}(k+1)$ & 439.80 & 432.29 & 7.51 & & 558.12 & 546.53 & 11.59 \\
\hline
\end{tabular}

Table 2 PED measurements for each model considered

\begin{tabular}{|c|c|c|c|c|c|c|}
\hline & \multicolumn{3}{|c|}{$k=3$} & \multicolumn{3}{|c|}{$k=5$} \\
\hline & PED & $\hat{D}_{e}$ & $\hat{p}_{\text {opt }}$ & PED & $\hat{D}_{e}$ & $\hat{p}_{\text {opt }}$ \\
\hline RJMCMC & 448.30 & 431.29 & 17.00 & 588.50 & 545.73 & 42.76 \\
\hline $\operatorname{MCMC}(k-1)$ & 642.81 & 486.45 & 156.36 & 585.34 & 563.43 & 21.91 \\
\hline $\operatorname{MCMC}(k)$ & 447.20 & 431.05 & 16.15 & 573.27 & 545.30 & 27.97 \\
\hline $\operatorname{MCMC}(k+1)$ & 452.14 & 432.26 & 19.88 & 579.22 & 546.11 & 33.11 \\
\hline
\end{tabular}

Table 3 Computational time in seconds spent in each models' fit

\begin{tabular}{ccccc}
\hline & RJMCMC & MCMC $(k-1)$ & MCMC $(k)$ & MCMC $(k+1)$ \\
\hline$k=3$ & 3.63 & 1.38 & 1.99 & 2.77 \\
$k=5$ & 3.02 & 1.97 & 2.31 & 2.76 \\
\hline
\end{tabular}

should have the best fit. It is possible to observe that, for both criteria, the model with $k$ fixed in the true value seems to fit the data better than its counterparts. However, the results are very similar, even when $k$ is estimated, increasing the size of the parametric vector, except when $k$ is fixed below the true value. The same conclusion is observed for both value fixed for $k$.

Table 3 shows the computational time in seconds spent in the fit of each model. As expected, RJMCMC requires more computational effort than its counterparts, although the difference is not so significant. Furthermore, the computational time increases as $k$ is fixed in a higher value, except when $k$ is estimate.

Thus, if the number of components is unknown and we use the MCMC algorithm to sample from the posterior distribution of the parametric vector, better results are attained by setting it greater than or equal to the true value. On the other hand, estimating the value of $k$ and using the RJMCMC method is a good alternative in this case, having similar performance to the case when we fixed $k$ at its true value. 
Table 4 Summary measurements for the point and $95 \%$ credibility interval estimates of the model parameters over 1,000 simulations considering k unknown (RJMCMC) and known (MCMC)

\begin{tabular}{lccccccccc}
\hline & $\mu_{1}$ & $\mu_{2}$ & $\mu_{3}$ & $\sigma_{1}^{2}$ & $\sigma_{2}^{2}$ & $\sigma_{3}^{2}$ & $w_{1}$ & $w_{2}$ & $w_{3}$ \\
\hline & & & & \multicolumn{7}{c}{ RJMCMC } \\
SRMSE & 0.10 & 0.48 & 0.45 & 0.11 & 0.81 & 0.38 & 0.05 & 0.05 & 0.08 \\
MAE & 0.08 & 0.36 & 0.22 & 0.07 & 0.63 & 0.18 & 0.05 & 0.04 & 0.05 \\
Cov. (\%) & 96.7 & 94.1 & 90.0 & 92.0 & 96.2 & 97.6 & 100.0 & 99.9 & 90.6 \\
Wid. & 0.45 & 1.96 & 0.84 & 0.39 & 4.08 & 1.23 & 0.16 & 0.22 & 0.25 \\
& & & & & MCMC & & & & \\
SRMSE & 0.10 & 0.48 & 0.14 & 0.11 & 0.96 & 0.18 & 0.05 & 0.06 & 0.03 \\
MAE & 0.08 & 0.36 & 0.11 & 0.07 & 0.70 & 0.15 & 0.05 & 0.04 & 0.03 \\
Cov. (\%) & 96.9 & 94.0 & 96.1 & 92.0 & 95.8 & 96.9 & 100.0 & 98.9 & 99.9 \\
Wid. & 0.45 & 1.96 & 0.59 & 0.40 & 4.19 & 0.81 & 0.16 & 0.23 & 0.25 \\
\hline & & & & & & & & &
\end{tabular}

Finally, we also generated 1,000 samples fixing the parameters at the previous values and obtained samples from the posterior distribution of the parametric vector, supposing $k$ known and fixed at the true value, 3, in the MCMC and estimating it using the RJMCMC algorithm. The estimates were then compared with the true values to evaluate the model's performance.

First, in $89.9 \%$ of the 1,000 samples the value of $k$ was correctly estimated when using RJCMC to sample from the posterior distribution. Table 4 shows summary statistics with some frequentist measures of the posterior distribution of the model parameters after reaching convergence. It reports the square root of the mean square error (SRMSE), the mean absolute error (MAE), the empirical nominal coverage of the $95 \%$ credibility intervals measured in percentages (Cov.) and the respective widths averaged over the 1,000 simulations (Wid.). In particular, the summary statistics of the components parameters are obtained conditioning on $k$ at the value with highest posterior probability.

The parameters are well estimated in both cases and the results are very similar considering each approach, except the parameters $\sigma_{2}^{2}$ and $\sigma_{3}^{2}$, which were slightly better estimated under the RJMCMC and MCMC approaches, respectively. The coverage of the $95 \%$ credibility intervals is close to the nominal level. These results indicate that similar results can be achieved considering $k$ unknown and fixing it at the true value. Although the MCMC algorithm has certain advantages with respect to computational cost compared to the RJMCMC, the number of components is generally unknown and estimating it can be a practical interest in the problem. Therefore, the RJMCMC is a reasonable alternative to sample from the posterior distribution in this case. 


\section{Application to a real dataset}

We applied the method to a real dataset that concerns antibody levels of Cytomegalovirus (CMV) in 5126 individuals, both males and females, from 6 years to 49 years old. This dataset was extracted from the 2003-2004 National Health and Nutrition Examination Survey (NHANES). ${ }^{1}$

The CMV is a member of the Herpesviridae family of viruses and according to Kusne, Shapiro and Fung (1999), it is a common virus that occurs widely throughout the population but rarely causes noticeable symptoms or significant health problems.

One method of detecting a CMV infection is by antibody testing of blood samples. This can also be used to determine if someone has had recent or past exposure. There are two types of CMV antibodies that are produced in response to a CMV infection, IgM and IgG, and one or both can be detected in the blood. IgM antibodies are the first to be produced by the body in response to a CMV infection and they are present in most individuals within a week or two after the initial exposure. On the other hand, IgG antibodies are produced by the body several weeks after the initial CMV infection and provide protection from primary infections. Levels of IgG rise during the active infection, then stabilize as the CMV infection resolves and the virus becomes inactive. After a person has been exposed to CMV, the person will have some measurable amount of CMV IgG antibodies in his/her blood for the rest of the lifetime. CMV IgG antibody testing can be used, along with IgM testing, to help confirm the presence of a recent or previous CMV infection. Particularly, this dataset consists of the IgG levels of CMV.

The range of values for the antibody levels CMV IgG are from 0.048 to 3.001. For the values reported as "out of range" (i.e., over the detectable range, $>3.00$ ) the survey specialists usually assign the value of 3.001. Thus, the observations are left-censored at 3.001 and there are many individuals with this particular value in the dataset. Figure 7 shows the antibody levels of CMV IgG distribution for 5126 individuals infected and not infected. The interest here is in identifying subgroups of $\operatorname{IgG}$ as a marker of the presence of the disease.

As shown in Figure 7, some heterogeneous subpopulations can be identified, so it is reasonable to fit the normal mixture model (2.1) to this dataset. However, in this particular case it is necessary to incorporate left-censoring. It is done assuming that we observe also $\left(l_{i}, u_{i}\right)$ associated to each $y_{i}$, for $i=1, \ldots, n$. Note that $y_{i}=l_{i}=u_{i}$ if the observation is not censored, $-\infty<l_{i}<u_{i}<\infty$ indicates

\footnotetext{
${ }^{1}$ Centers for Disease Control and Prevention (CDC). National Center for Health Statistics (NCHS). National Health and Nutrition Examination Survey Data. Hyattsville, MD: U.S. Department of Health and Human Services, Centers for Disease Control and Prevention [2003-2004] [http://www. cdc.gov/nchs/nhanes].
} 


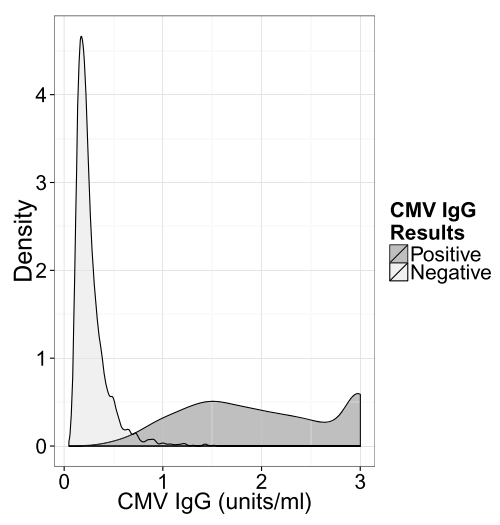

Figure 7 Distribution of antibody levels of CMV IgG (units/ml) for 5126 individuals infected by the virus or not.

interval-censored observation, $-\infty<l_{i}<u_{i}=\infty$ indicates right-censored observation and $-\infty=l_{i}<u_{i}<\infty$ indicates left-censored observation, which is our particular case. It will be treated in the inference, through the likelihood function, which will be written now including the censor to the expression (2.2) in the following way:

$$
p(\mathbf{y} \mid \boldsymbol{\Phi})=\prod_{i=1}^{n} \int_{l_{i}}^{u_{i}} \sum_{j=1}^{k} w_{j} f\left(y_{i} \mid \boldsymbol{\theta}_{j}\right),
$$

with the convention that $\int_{l}^{u} f(y) d y=f(l)=f(u)$ whenever $l=u$ (uncensored observation).

Regarding inference, we also considered both estimating $k$ and fixing it on three different values, that are 2, 3 and 4. We worked with a subsample of size 1,000 selected from the complete data. For the RJMCMC and MCMC simulations, we generated 50,000 samples from the posterior distribution, discarded the first 10,000, then thinned the chain by taking every 10th sample value. Figures 9, 10, 11 and 12 in Appendix B presents the trace plot with the posterior distribution of the components of $\boldsymbol{\mu}$ and $\boldsymbol{\sigma}^{2}$ for each MCMC and RJMCMC simulations. Analyzing them leads us to conclude that convergence seems to be reached for all the parameters.

Figure 8 displays the posterior distribution of $k$ and predictive densities of antibody levels when estimating $k$ (RJMCMC) and fixing it (MCMC), represented by the dashed and dotted lines, respectively. The posterior distribution of $k$ obtained from RJMCMC simulation favors 4 components. The predictive plots for RJMCMC and MCMC with $k$ fixed in 4 are very similar, showing good performance even when $k$ is estimated.

Table 5 presents the value of $\mathrm{DIC}_{3}$ and PED for each approach considered in this study. Note that, different from previous studies, the model with $k$ unknown 

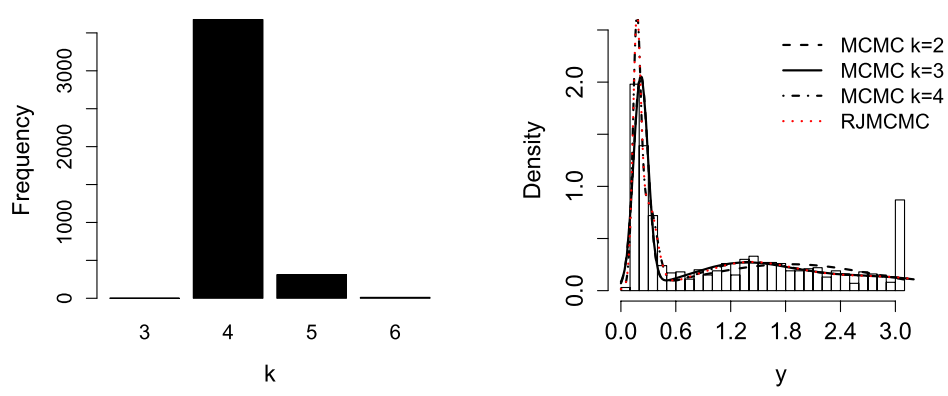

Figure 8 Posterior distribution of $k$ and predictive densities for the real dataset.

Table 5 DIC and PED measurements for the models considered in the real dataset

\begin{tabular}{lcccccc}
\hline & DIC & $\bar{D}$ & $p_{D}$ & PED & $\hat{D}_{e}$ & $\hat{p}_{\text {opt }}$ \\
\hline RJMCMC & 1826.97 & 1784.97 & 42.00 & 1731.00 & 1696.45 & 34.55 \\
MCMC $(k=2)$ & 1956.46 & 1912.94 & 43.52 & 1856.94 & 1845.74 & 11.19 \\
MCMC $(k=3)$ & 1916.36 & 1876.77 & 39.59 & 1801.89 & 1774.89 & 27.01 \\
MCMC $(k=4)$ & 1830.53 & 1788.18 & 42.35 & 1729.87 & 1697.75 & 32.12 \\
\hline
\end{tabular}

presents smaller DIC than the approach which considered $k$ fixed in 4, although PED be smaller for this one. Thus, using a censored dataset, we observe more benefits in considering $k$ unknown and using RJMCMC.

\section{Conclusions and suggestions for future work}

We considered the problem of the fit of mixture models for heterogeneous populations under different levels of heterogeneity. We concluded that in some cases it is not necessary to choose other relabeling algorithms, as described in Stephens (2000), in order to improve the label-switching problem. To assign a weakly informative prior distribution for the mixture proportions, even for more homogeneous populations, was efficient in many cases.

We also evaluated the inference of the model when the number of components is unknown and RJMCMC maybe used and when it is fixed at a known value. We concluded that when the number of mixture components is unknown, the RJM$\mathrm{CMC}$ is a feasible alternative, achieving similar results when this number is fixed at the true value. Nevertheless, it requires slightly greater computational effort than MCMC. On the other hand, when not interesting in estimating this number, setting it at a value smaller than the true one will generate poor estimates, although, similar results are obtained when fixing it at the true value or greater than this. We also studied the frequentist properties of the Bayes estimators obtained from the fits, through a simulation study and we also observed similar results in both approaches. 
We applied the methodology to a left-censored real dataset with antibody levels of Cytomegalovirus (CMV) in individuals. We concluded here that estimating the value of $k$ was necessary because the distribution of the dataset does not provide much information about 4 subgroups. Thus, it would be possible here to fix $k$ in a small value, and to underestimate the predictive densities. Furthermore, different from previous studies, in this case, DIC indicates as the best model the one with $k$ unknown. So, we suggest in problems with censored dataset the use of RJMCMC.

Finally, the main findings of this work encourage an extension of this study to other mixture distributions, as the Poisson model discussed in Viallefont, Richardson and Green (2002).

\section{Appendix A: $R$ code to perform the experiments}

All the previous experiments were done using the R package mixAK. Follow we have the main code needed to reproduce the research paradigm for $\boldsymbol{\mu}$ and $\boldsymbol{\sigma}^{2}$. To reproduce it for $\mathbf{w}$, follow the same steps considered for $\sigma^{2}$.

\section{A.1 $R$ code to reproduce studies in Section 3}

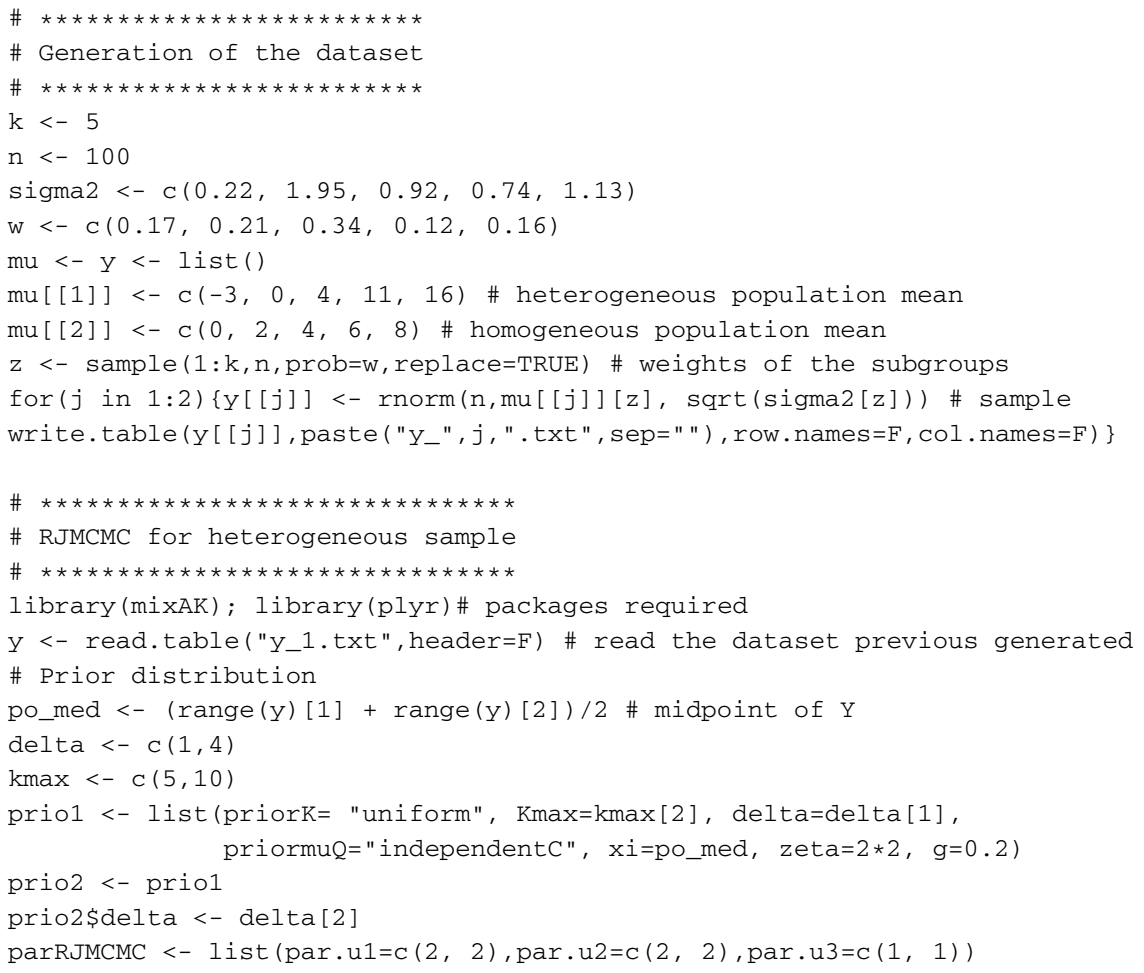




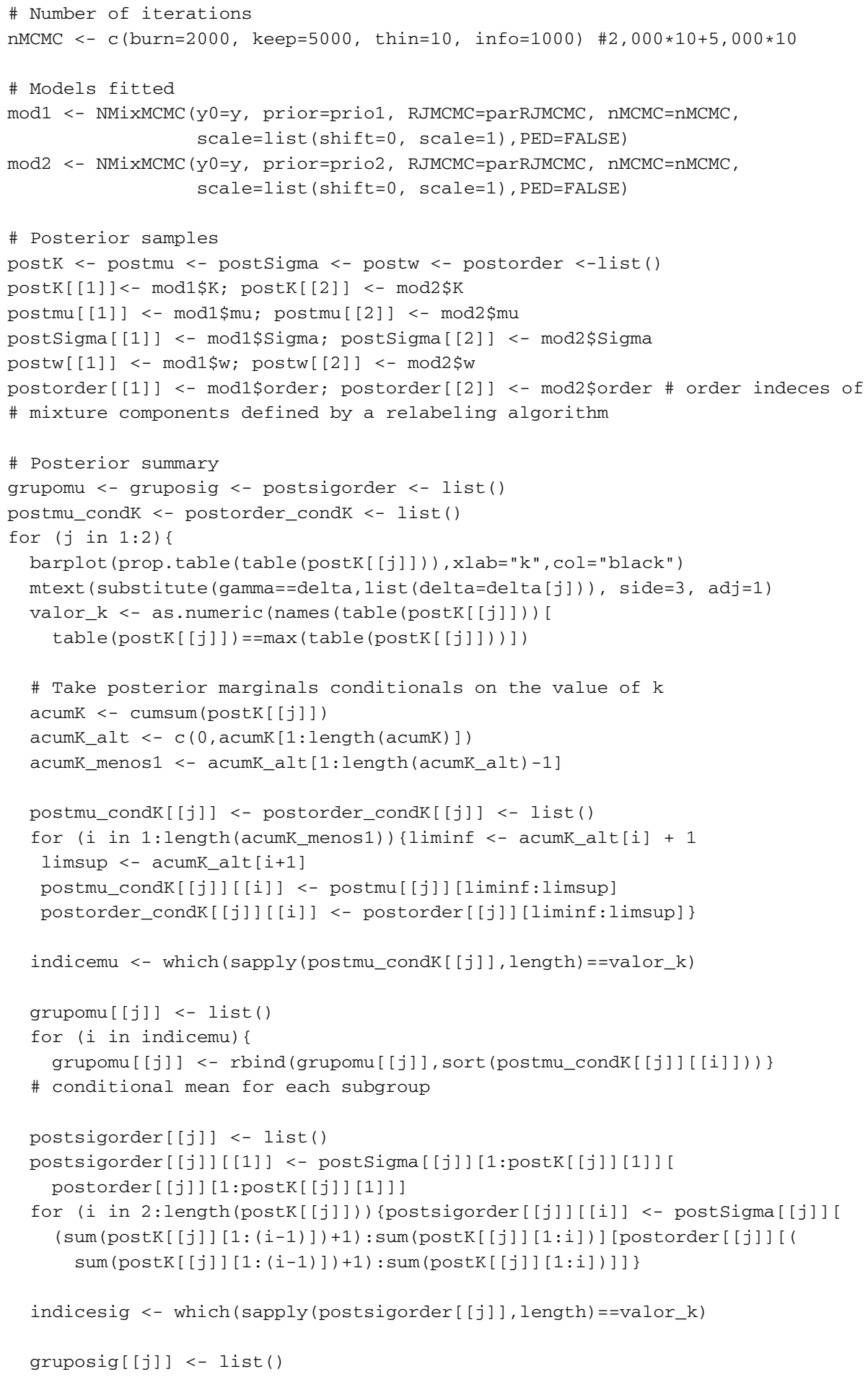


for (i in indicesig)\{gruposig[[j]] <- rbind(gruposig[[j]], postsigorder[[j]][[i]])\} \# conditional variance for each subgroup \}

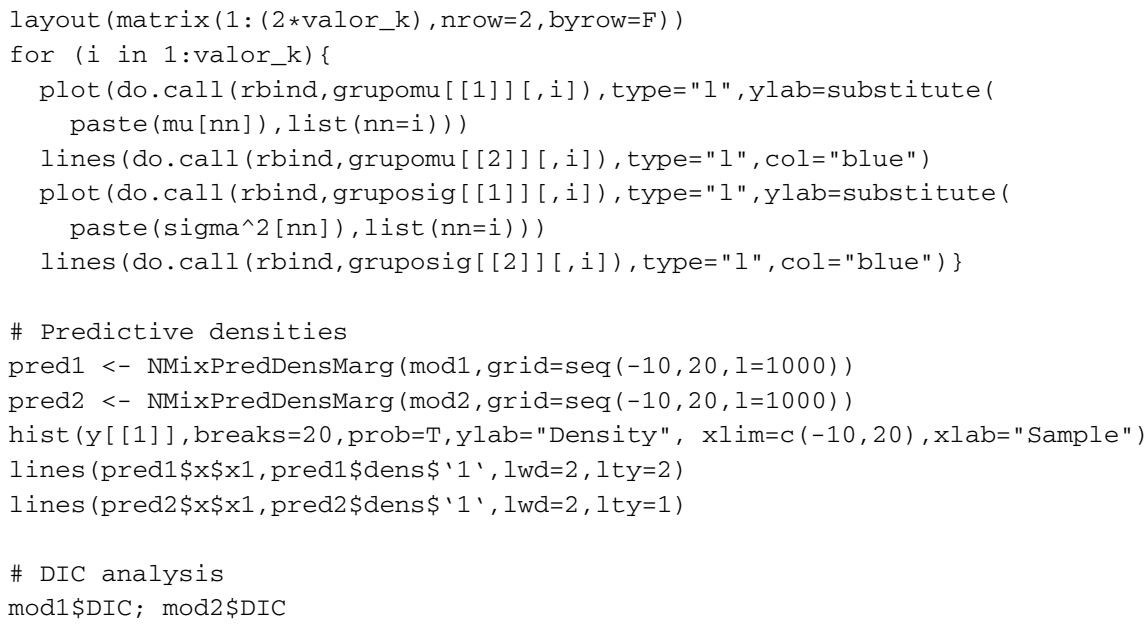




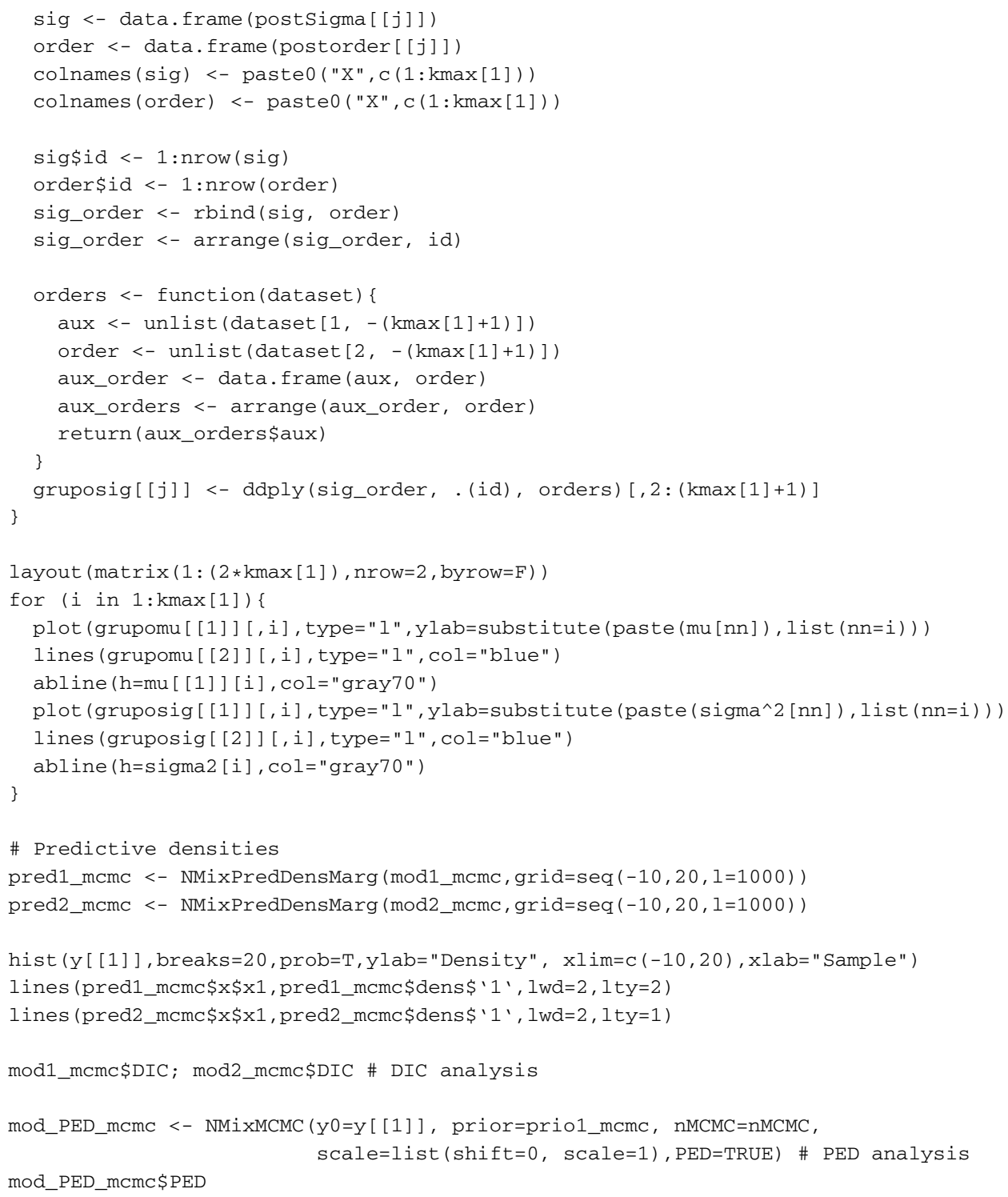

\section{A.2 $R$ code to reproduce studies in Section 4}

library (foreign)

download.file("http: / /wwwn.cdc.gov/Nchs/Nhanes /2003-2004/SSCMV_C.XPT" , "download_SSCMV_C.XPT", mode="wb" )

y <- sample (read.xport ( "download_SSCMV_C.XPT") \$SSCMVOD, 1000)

censor <- rep (1, length (y))

censor $[$ which $(y==3.001)]<-0$ 


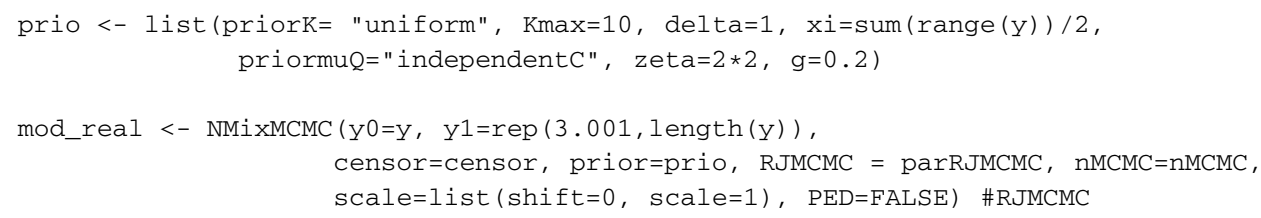

\section{Appendix B: Assessment of MCMC and RJMCMC with real data}

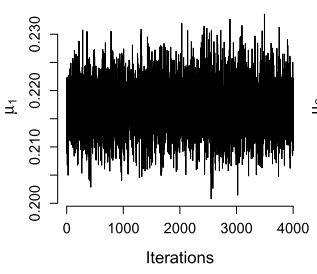

(a) $\mu_{1}$

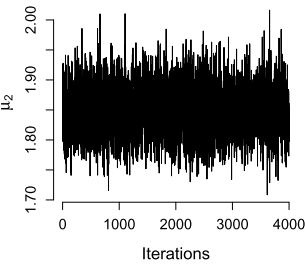

(b) $\mu_{2}$

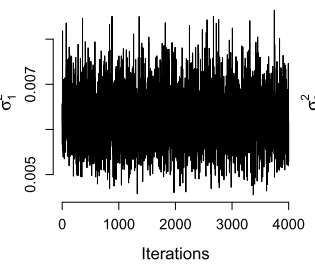

(c) $\sigma_{1}^{2}$

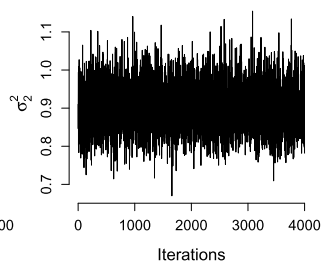

(d) $\sigma_{2}^{2}$

Figure 9 Trace plots with the posterior densities of the parameters $\boldsymbol{\mu}$ and $\boldsymbol{\sigma}^{2}$ obtained from the fit of the normal mixture model assuming $k$ to be known and fixed at the value 2 in the real dataset (MCMC).

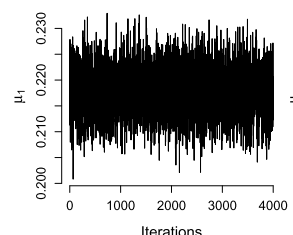

(a) $\mu_{1}$

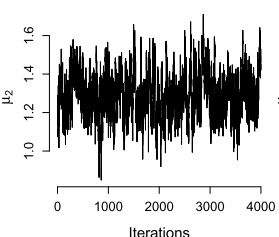

(b) $\mu_{2}$

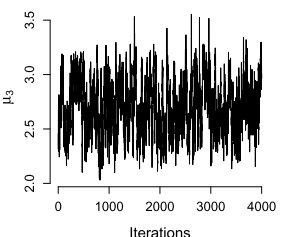

(c) $\mu_{3}$

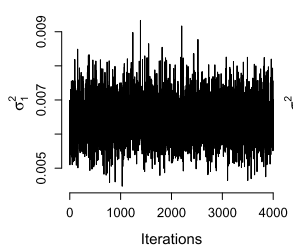

(d) $\sigma_{1}^{2}$

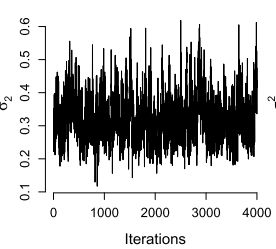

(e) $\sigma_{2}^{2}$

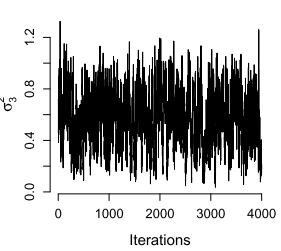

(f) $\sigma_{3}^{2}$

Figure 10 Trace plots with the posterior densities of the parameters $\boldsymbol{\mu}$ and $\boldsymbol{\sigma}^{2}$ obtained from the fit of the normal mixture model assuming $k$ to be known and fixed at the value 3 in the real dataset $(M C M C)$. 


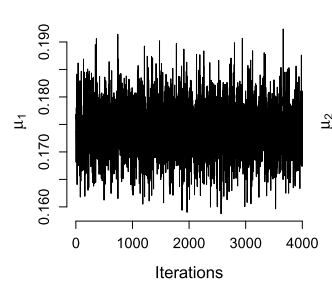

(a) $\mu_{1}$

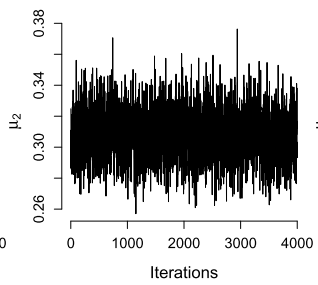

(b) $\mu_{2}$

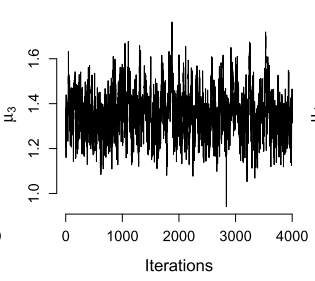

(c) $\mu_{3}$

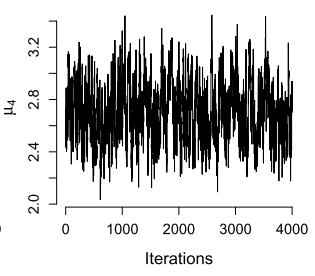

(d) $\mu_{4}$

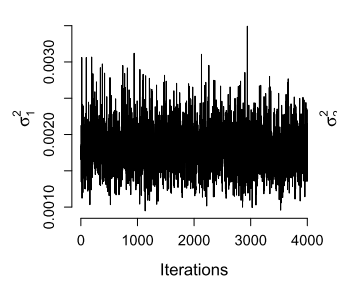

(e) $\sigma_{1}^{2}$

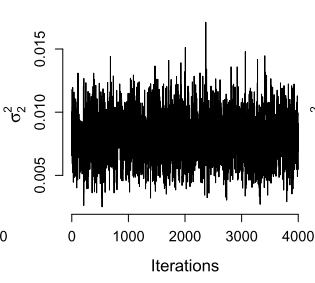

(f) $\sigma_{2}^{2}$

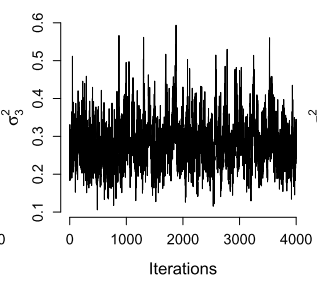

(g) $\sigma_{3}^{2}$

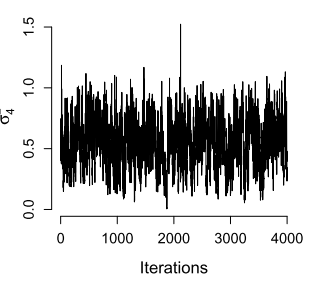

(h) $\sigma_{4}^{2}$

Figure 11 Trace plots with the posterior densities of the parameters $\boldsymbol{\mu}$ and $\boldsymbol{\sigma}^{2}$ obtained from the fit of the normal mixture model assuming $k$ to be known and fixed at the value 4 in the real dataset $(M C M C)$.

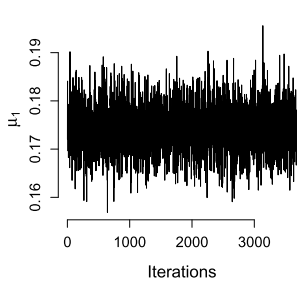

(a) $\mu_{1}$

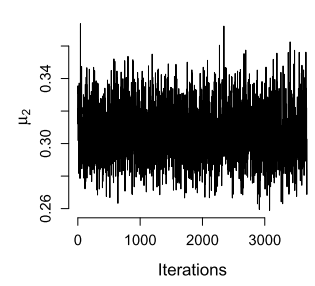

(b) $\mu_{2}$

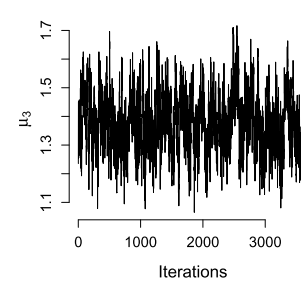

(c) $\mu_{3}$

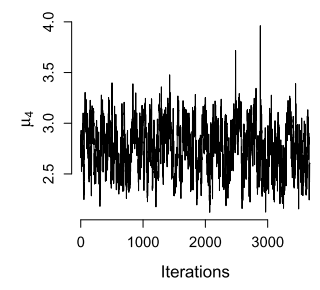

(d) $\mu_{4}$

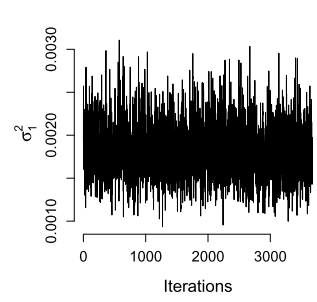

(e) $\sigma_{1}^{2}$

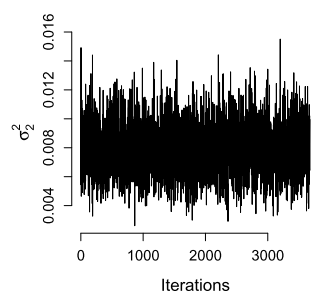

(f) $\sigma_{2}^{2}$

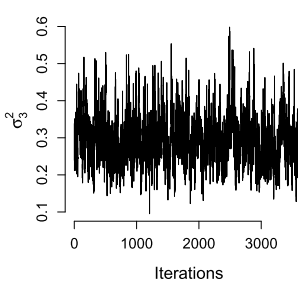

(g) $\sigma_{3}^{2}$

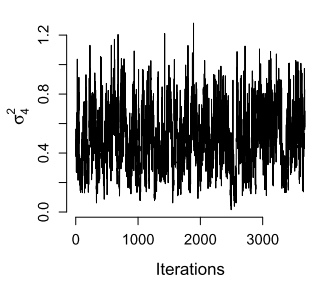

(h) $\sigma_{4}^{2}$

Figure 12 Trace plots with the posterior densities of the parameters $\boldsymbol{\mu}$ and $\boldsymbol{\sigma}^{2}$ obtained from the fit of the normal mixture model assuming $k$ to be unknown in the real dataset (RJMCMC). 


\section{Acknowledgments}

The authors thank the Associate Editor and the referees for many valuable comments and suggestions.

\section{References}

Celeux, G., Forbes, F., Robert, C. P., Titterington, D. M., et al. (2006). Deviance information criteria for missing data models. Bayesian analysis 1, 651-673. MR2282197

Dellaportas, P. and Papageorgiou, I. (2006). Multivariate mixtures of normals with unknown number of components. Statistics and Computing 16, 57-68. MR2224189

Diebolt, J. and Robert, C. P. (1994). Estimation of finite mixture distributions through Bayesian sampling. Journal of the Royal Statistical Society. Series B (Methodological) 56, 363375. MR1281940

Green, P. J. (1995). Reversible jump Markov chain Monte Carlo computation and Bayesian model determination. Biometrika 82, 711-732. MR1380810

Jasra, A., Holmes, C. C. and Stephens, D. A. (2005). Markov chain Monte Carlo methods and the label switching problem in Bayesian mixture modeling. Statistical Science 20, 50-67. MR2182987

Jordan, M. I. (2004). Graphical models. Statistical Science 19, 140-155. MR2082153

Komárek, A. (2009). A new R package for Bayesian estimation of multivariate normal mixtures allowing for selection of the number of components and interval-censored data. Computational Statistics \& Data Analysis 53, 3932-3947. MR2744295

Kusne, S., Shapiro, R. and Fung, J. (1999). Prevention and treatment of cytomegalovirus infection in organ transplant recipients. Transplant infectious disease 1, 187-203.

McLachlan, G. and Peel, D. (2004). Finite Mixture Models. Wiley. MR1789474

Nobile, A. (2004). On the posterior distribution of the number of components in a finite mixture. Annals of statistics 32, 2044-2073. MR2102502

Plummer, M. (2008). Penalized loss functions for Bayesian model comparison. Biostatistics 9, 523539.

R Core Team (2014). R: A Language and Environment for Statistical Computing. R Foundation for Statistical Computing. Vienna, Austria. Available at http://www.R-project.org/.

Redner, R. A. and Walker, H. F. (1984). Mixture densities, maximum likelihood and the EM algorithm. SIAM review 26, 195-239. MR0738930

Richardson, S. and Green, P. J. (1997). On Bayesian analysis of mixtures with an unknown number of components. Journal of the Royal Statistical Society. Series B (Methodological) 59, 731792. MR1483213

Roeder, K. and Wasserman, L. (1997). Practical Bayesian density estimation using mixtures of normals. Journal of the American Statistical Association 92, 894-902. MR1482121

Spiegelhalter, D. J., Best, N. G., Carlin, B. P. and Van Der Linde, A. (2002). Bayesian measures of model complexity and fit. Journal of the Royal Statistical Society: Series B (Statistical Methodology) 64, 583-639. MR1979380

Stephens, M. (2000). Dealing with label switching in mixture models. Journal of the Royal Statistical Society: Series B (Statistical Methodology) 62, 795-809. MR1796293

Viallefont, V., Richardson, S. and Green, P. J. (2002). Bayesian analysis of Poisson mixtures. Journal of nonparametric statistics 14, 181-202. MR1905593

Escola Nacional de Ciências Estatísticas

Rio de Janeiro, RJ

Brazil

E-mail: carolinavalanic@gmail.com
Departamento de Métodos Estatísticos Universidade Federal do Rio de Janeiro Rio de Janeiro, RJ

Brazil

E-mail: kelly@dme.ufrj.br 